\title{
Generic rule-sets for automated detection of urban tree species from very high- resolution satellite data
}

\begin{abstract}
The sustainable management and monitoring of urban forests is an important activity in the urbanized world, and operational approaches require information about the status of urban trees to determine the best strategy. One limitation in urban forest studies is the detection and discrimination of tree species using limited training data. Thus, this study focuses on developing generic rule sets from high-resolution WorldView-2 imagery in conjunction with spectral, spatial, colour and textural information for automated urban tree species detection. The object-based image analysis and its combination with statistical analysis of object features is utilized for this purpose. Results of attribute selection indicated that from 55 attributes, only 26 were useful to discriminate urban tree species, namely Messua ferrea L., Samanea saman and Casuarina sumatrana. Finally, the high overall accuracy, approximately $86.87 \%$ with kappa of 0.75 confirmed the transferability of the generic model.
\end{abstract}

Keyword: WorldView-2; Object-based image analysis; CfsSubsetEval; Urban tree species 
